syndromes, has recently been reported in a family with atypical Panyiotopoulos syndrome (Grosso S et al. Neurology 2007;69:609-611).

\title{
GOURMAND SYNDROME IN A CHILD WITH EPILEPSY
}

A preoccupation with food, increased appetite and a preference for fine food had developed in a 10-year-old obese boy soon after the onset of refractory seizures at 8 years of age, in a report from University Hospital of Geneva, Switzerland. He had streptococcal B sepsis and hemorrhage in the right temporoparietal lobes during the neonatal period. Seizures were stereotyped, with loss of contact and oral automatisms or dystonic posturing of the left hand. Their frequency was once a week to 7 times a day. They were uncontrolled despite trial of 5 different antiepileptic drugs. He preferred to cook meals for himself rather than eat at fast food restaurants. He had no history of emotional disorder, binge eating, bulimia, or preoccupation with his weight. Neurological examination revealed a left inferior quadrantanopia and left dysdiadochokinesia. In presurgical evaluation, the EEG showed a right posterior focus, and diffuse bilateral parasaggital seizure activity in sleep. MRI revealed a porencephaly, periventricular gliosis and hemosiderin deposits in the right parietal lobe. Neuropsychological examination showed visuospatial memory deficits, discrete signs of neglect, more pronounced in the postictal phase, with perseveration and confabulation. (Kurian M, Schmitt-Mechelke T, Korff C, Delavelle J, Landis T, Seeck M. "Gourmand syndrome" in a child with pharmacoresistant epilepsy. Epilepsy Behav August 2008;13:413415). (Respond: Dr M Kurian, Presurgical Epilepsy Evaluation Unit, Department of Neurology, University Hospital of Geneva, 24 rue Micheli-du-Crest, 1211 Geneva 14, Switzerland. E-mail: mary.kurian(ahcuge.ch).

COMMENT. "Gourmand" syndrome" reported in adults with right hemisphere lesions is often associated with epilepsy, and the eating disorder may be reversible when epilepsy is controlled. (Levine R et al. Epilepsy Behav 2003;4:781-3; cited by Kurian et al). The authors recommend brain imaging to rule out right hemisphere lesion in a patient who develops disturbed eating habits following a head injury or seizures.

\section{RISK OF DROWNING IN EPILEPSY}

The risk of drowning in patients with epilepsy is quantified by a meta-analysis of published reports, in a study at the Institute of Neurology, Queen Square, London UK. The number of deaths from drowning and the number of person-years at risk were estimated in 51 cohorts of people with epilepsy. Standardized mortality ratios (SMRs) (observed deaths divided by the expected deaths) were calculated for each cohort and for the total population. Compared with 4.7 expected deaths, 88 drowning deaths were reported in people with epilepsy, giving an SMR of 18.7 (95\% Cl 15-23). The 51 cohorts combined had 206,596 patient-years of follow-up. In people with epilepsy and learning disability, the SMR was 25.7, and in those in institutional care, 96.9. In those with temporal lobectomy for epilepsy, the SMR was 41.1. Using National Registries for estimation of drowning deaths in people with epilepsy in England and Wales (1999-2000), the SMR was 15.3. (Bell GS, Gaitatzis A, Bell CL, Johnson AL, Sander JW. Drowning in people with epilepsy. How great is the risk. Neurology Aug 19 2008;71:578-582). (Reprints: Prof Ley Sander, Box 29, Department of 